\title{
"CERTUM TESTIMONIUM»: ATTI NOTARILI E RELAZIONI MERCANTILI-FINANZIARIE (ASTI E PIEMONTE MERIDIONALE SECOLI XIII-XIV)*
}

\author{
"CERTUM TESTIMONIUM»: NOTARIAL ACTS AND MERCANTILE- \\ FINANCIAL RELATIONS (ASTI AND SOUTHERN PIEDMONT, \\ 13TH-14TH CENTURIES)
}

\author{
Ezio Claudio Pia
}

CRISM (Centro di Ricerca sulle Istituzioni e le Società Medievali) presso il Dipartimento di Studi Storici dell'Università di Torino

\begin{abstract}
English: The notarial certification of financial and mercantile relations is one of the aspects of what Paolo Cammarosano has described as the "new weight of the notaries in the complex of political and social life» from the twelfth century onwards, an evolution characterised by a change in the «borders between public and private».

The aim of this paper is to understand, in the concreteness of documentary production, the role of notaries in defining the modes and forms of economic relations. The analysis will focus on examples taken from documents of the 13th and 14th centuries relating to Asti and southern Piedmont.

The cases presented allow a comparison between writing practices - characterised by a marked experimentation - and articulated credit relations.

In this way it is possible both to understand the socio-economic dynamics that innervate one of the main municipalities of northern Italy and to highlight the functionality of certification processes in the hands of notaries, who exercised a notable role of orientation in financial and commercial relations.

This analysis cannot prescind from the historiographic lines drawn by the generous magisterium of Gian Giacomo Fissore, to whom we owe decisive studies on subalpine notaries and particular attention to the notarial registers of Asti in the 13th and 14th centuries.
\end{abstract}

These registers collect instrumenta concerning juridical transactions - mortgages, sales, rents - through which a network of relationships is outlined, involving the city and the territory, a large sector of central-southern Piedmont gravitating on the municipality and diocese of Asti. In particular, the cases that we will examine concern the financial activity of laymen and clergymen - covering different types of credit, from financing to the management of investments, such as deposits and commende - and the definition

* Italian Review of Legal History, 7 (2021), n. 18, pagg. 645-659

* https://riviste.unimi.it/index.php/irlh/index

* ISSN 2464-8914 - DOI 10.54103/2464-8914/16903. Articolo pubblicato sotto Licenza CC-BY.

* Desidero ringraziare per la preziosa occasione di confronto Paolo Cammarosano e Marialuisa Bottazzi, coordinatori del Progetto "Notariato medievale italiano", organizzato dal CERM di Trieste, dal Centro Studi Santa Rosa da Viterbo (presieduto da Attilio Bartoli Langeli) e dalla Scuola di Paleografia e Storia di Viterbo. 
of give-and-take relationships, carried out both informally and through a judicial process. The attested documentary forms therefore reveal a complex system of regulation, managed by experts in judicial and economic mediation, sanctioned by notaries and capable of a widespread work of remodelling the internal dynamics of society. It is evident that the notary's intervention defines the nodal elements of economic relations, of which he grasps the criticalities that the writing attempts to resolve by diluting the traits of uncertainty in the establishment of credit relations, constructing mechanisms of compensation and regulation, and again elaborating the ways and forms of the complex encounter between credit and politics in the case of fiscal default. A role that Rolandino effectively outlines, "tabelliones vel scrinearios appellamus, eo quod sicut inventum thessaurum in scrineo reconditur ut servetur, sic merito debet omnis fides omnisque legalitas et quicquid eis in fide ac legalitate committitur per eos inviolabiliter reservari", describing a "treasure" built on fides and legalitas, inviolably preserved thanks to notarial skills.

Not only that: "omnibus que [tabelliones] scripserint fides datur, nec audet quis de levi ausu temerario infringere publice quod scripserint». The fides fixed in a scripture cannot therefore be "broken". In this perspective, a "treasure trove" is being built up through writing, framing relationships characterised by a marked dynamism and which - precisely in order to respond to their structural instability - in turn takes on dynamic traits, regulating the projection in time of obligations and commitments.

The lasting functionality of potentially conflicting relationships is at stake in the deeds, since the documents notarized by the notaries do not simply sanction renegotiation but also set out the mechanisms that ensure its effectiveness in a diachronic perspective. These are articulated practices that correspond to precise competences of the notary, who, according to the widespread thirteenth-century formulary of the jurist Martino da Fano, is the depositary of a peculiar ability to discernere: "discretus, in intelligendo, disquirendo et componendo».

Keywords: notaries; credit; taxation; interest rates; 13 th and 14th centuries

Abstract Italiano: La documentazione astigiana dei secoli XIII e XIV restituisce la sperimentazione, da parte del notariato, di modi e forme di definizione dei rapporti economici, segno - come scrive Paolo Cammarosano - del «peso dei notai nel complesso della vita politica e sociale». La scrittura notarile inquadra le criticità delle relazioni mercantili-finanziarie, costruendo meccanismi di compensazione e di regolazione, e intervenendo nel complesso incontro tra credito e politica in ambito fiscale. Una conferma della peculiare competenza «in intelligendo, disquirendo et componendo» che il giurista duecentesco Martino da Fano attribuisce al notariato.

Parole chiave: notai; credito; fiscalità; tasso di interesse; secoli XIII e XIV

Sommario: 1. Introduzione - 2. «Pecunia pretaxata»: fissazione preliminare del debito 3. "Aliqua alia instrumenta»: un debito definito in itinere - 4. Cessione e rinegoziazione del credito -5 . Sperimentazioni nel contenzioso fiscale -6 . Conclusioni 


\section{Introduzione}

La certificazione notarile di relazioni finanziarie e mercantili è, secondo Paolo Cammarosano, uno degli aspetti del «nuovo peso dei notai nel complesso della vita politica e sociale» a partire dal XII secolo, esito di un mutato assetto delle «frontiere tra pubblico e privato» ${ }^{1}$. Una crescente centralità della definizione dei rapporti economici in capo al notariato efficacemente esplicitata nei quattrocenteschi Statuti del Collegio dei Notai genovesi, secondo i quali «ars notariorum, que in scripturarum fide omnem fidem superat, (...) est humanorum negotiorum et in vita et in morte ac post mortem certum testimonium ${ }^{2}$.

L'indagine oggetto del presente intervento è rivolta alla documentazione di pieno Duecento e di inizio Trecento relativa ad Asti e all'ampio settore compreso tra Piemonte centrale e Liguria che all'epoca faceva capo al comune astigiano: un quadro che consente sia di delineare gli sviluppi - colti anche in fase di elaborazione - dei rapporti socio-economici che innervano uno dei principali organismi politico-territoriali dell'Italia settentrionale ${ }^{3}$, sia di mettere in luce il ruolo di orientamento nelle dinamiche finanziarie e commerciali esercitato dai notai.

Questo percorso non può prescindere dalle linee storiografiche tracciate dal generoso magistero di Gian Giacomo Fissore cui si debbono studi decisivi sul notariato subalpino e una particolare attenzione alla documentazione in registro astigiana dei secoli XIII e XIV4. Indagini che permettono di cogliere il nesso tra pratiche scrittorie e dinamiche economiche: il carattere promiscuo dei registri nei quali entro una cronologia breve trovano definizione rapporti variegati, con una molteplicità di autori e di forme contrattuali - restituisce il progressivo consolidarsi del ruolo dei notai ${ }^{5}$. A tali contenitori "promiscui" si attaglia la categoria di "ibridazione", proposta da Fissore per interpretare la prismatica attività del notariato ${ }^{6}$ : si assiste infatti a un intreccio di competenze - notarili in senso stretto e cancelleresche al servizio della curia - per una committenza a sua volta "mista", in quanto costituita di laici ed ecclesiastici, con rogatari - infine - anch'essi connotati da un duplice inserimento, in quanto possono essere sia laici sia chierici .

Tale pluralità di relazioni segna la pervasività dei lessici dei quali i notai sono depositari e la funzionalità politica ed economica delle pratiche che trovano

\footnotetext{
${ }^{1}$ Cammarosano, 1991, p. 268.

2 Puncuh (ed.), 1966, p. 303.

3 Bordone, 1980; Pia, 2017. È opportuno ricordare la proiezione internazionale dell'economia astigiana, come indica il durevole ruolo dei mercanti-banchieri originari di Asti e di zone limitrofe nella finanza europea: cfr. Bordone (ed.), 1994a; Bordone, 1994b, pp. 1-17; Bordone, 2005, pp. 14-16; una lettura di sintesi in Pia, 2013, pp. 817-840.

4 Fissore, 1973, pp. 416-510; Fissore, 1977; Fissore, 1995, pp. 281-304; Fissore, 2003, pp. 365-414; Fissore, 2009, pp. VIII-XV; Fissore, 2013, pp. 25-59.

${ }^{5}$ Cammarosano, 1991, pp. 270-271.

${ }^{6}$ Fissore, 2003, pp. 395 (nota 102) e 396.

${ }^{7}$ Cfr. Pia, 2010, pp. 119-125.
} 
una mediazione e una sistemazione appunto nella produzione notarile ${ }^{8}$ : si può dunque rilevare, riprendendo una considerazione di Attilio Bartoli Langeli, che a «farsi fonte sono non soltanto i contenuti oggettivi dei documenti ma la documentazione stessa in quanto risultato di una prassi (...), di una strategia» ${ }^{9}$. Un nesso strutturale, quello tra la certificazione del notaio - «persona privilegiata ad negotia hominum publice, et authentice conscribenda ${ }^{10}$, secondo la nota definizione di Rolandino - e l'intensificarsi delle relazioni economiche, riconoscibile su scale differenti, in solide realtà urbane ma anche in contesti rurali. Come illustra Vito Piergiovanni,

partendo da questa situazione relativa ai soggetti ed alle tecniche che mutano con la rivoluzione commerciale dei secoli XI e successivi, è il quadro ambientale ed operativo che si arricchisce e si complica per arrivare ad essere quello in cui opera Rolandino. Lo spazio urbano, i nuovi assetti istituzionali, una diversa organizzazione politica e sociale cambiano in maniera radicale la figura del notaio. La civiltà urbana e mercantile lo assume come elemento di cerniera tra la dottrina e la pratica e lo induce ad aggiornare il proprio modo di essere rapportandosi ai soggetti protagonisti del nuovo momento storico ${ }^{11}$.

Se il percorso di Rolandino appare radicato nel vivace quadro politico-economico bolognese, non meno rilevante risulta l'attività del notariato nella fissazione di estesi rapporti creditizi nell'ambiente rurale - Crédit rural et endettement paysan è il titolo di uno status quaestionis sulle aree piemontese, lombarda e toscana tra $\mathrm{XI}$ e XIV secolo ${ }^{12}$-, rapporti dai quali emerge la concretezza di relazioni economiche legate ai cicli della produzione agricola, a conferma di una diffusa opera di certificazione.

Un legame molto stretto unisce d'altro canto le città al contado, come testimoniano i registri astesi che raccolgono instrumenta relativi a negozi giuridici - mutui, compravendite, affitti - mediante i quali si delinea una rete di relazioni che investe la città e l'estesa area del Piemonte centro-meridionale gravitante sul

\footnotetext{
${ }^{8}$ L'analisi dei processi documentari di cui stiamo trattando si lega a quella relativa al rapporto tra Chiesa e città: a questo proposito, Giorgio Chittolini ha osservato come i notai italiani, in quanto depositari di una specifica auctoritas, diventino elementi nodali della "piena integrazione delle istituzioni vescovili (...) [nel] mondo urbano e comunale» (Chittolini, 1994, p. 225); a sua volta Gian Giacomo Fissore (cfr. sopra nota 5), mediante I'analisi della documentazione vescovile o capitolare subalpina e in particolare astigiana, ha delineato il progressivo sviluppo di una burocrazia di curia incentrata sui chierici notai, una visione riconducibile a quella tendenza a "elaborazione intellettuale, definizione giuridica, disciplinamento e riorganizzazione» che, come ha messo in luce Antonio Olivieri, accomuna le istituzioni cittadine e quelle ecclesiastiche (Olivieri, 2003, p. 703).

${ }^{9}$ Bartoli Langeli, 2004a, p. 8.

10 Rolandini, 1565, p. 789.

${ }^{11}$ Piergiovanni, 2012a, p. 1423

12 Gaulin, Menant, 1998, pp. 37-67; cfr. anche Menant, Redon, 2004.
} 
comune e sulla diocesi di Asti ${ }^{13}$. In particolare il quadro che esamineremo riguarda sia l'attività finanziaria di laici ed ecclesiastici - che copre tipologie diverse di credito, dai finanziamenti alla gestione di veri e propri investimenti, quali depositi e commende - sia la definizione dei rapporti di dare-avere, realizzata sul piano informale oppure mediante un iter giudiziario.

Le forme documentarie attestate rivelano pertanto un sistema di regolazione sancito dai notai e capace di un ricorrente rimodellamento delle dinamiche interne alla società. Relazioni diffuse, spesso radicate in una zona grigia nella quale l'opera del notaio si situa sul confine della duplice appartenenza alle strutture comunali ed ecclesiastiche, condizione che ne connota l'attività professionale nell'Italia centro-settentrionale tra la fine del XII secolo e il XIV, come rivelano i casi di Siena, di Milano e di Verona, prima che si avvii una graduale specializzazione delle competenze rispetto allo status dei committenti ${ }^{14}$.

\section{2. «Pecunia pretaxata»: fissazione preliminare del debito}

Nei registri due-trecenteschi astesi, numerose relazioni di dare-avere sono inquadrate nelle carte di mutuo rogate da notai e riconducibili a due modelli distinti:

- nel primo compare l'indicazione della somma o del quantitativo di materia prima da restituire a fronte di un credito iniziale non esplicitato;

- nel secondo è presente la dichiarazione della somma o della quantità (espressa in misure di peso o di capacità) prestate.

In entrambi i casi viene fissato il termine della restituzione, cui seguono l'obbligo di corrispondere spese, danni e interessi in caso di inosservanza e impegni volti a ridurre le possibilità per il debitore di avvalersi di tutele legali $i^{15}$.

L'elemento nodale, nella costruzione delle carte di mutuo, è dunque la mancata esplicitazione del rapporto tra la quantità di denaro e di merce prestati e l'ammontare di quanto va reso. Sia nelle carte che omettono l'importo del credito sia in quelle che lo dichiarano, il riferimento agli interessi - quando presente - riguarda solo la fase successiva alla scadenza dei termini: non compare alcun cenno al costo dell'operazione, cioè ai tassi applicati nel periodo che precede la messa in mora del debitore. Alcune attestazioni indirette, tuttavia, permettono di

13 Cfr. sopra nota 4.

${ }^{14}$ Chironi, 2005; Belloni, Lunari, 2004, pp. XVII e sgg; Varanini, Gardoni, 2009, pp. 241272.

${ }^{15}$ Fino agli anni Sessanta del XIII secolo, compaiono entrambi i formulari, con una prevalenza di atti che omettono l'ammontare del prestito mediante la generica dizione "tot ex suis denariis», mentre questo modello risulta quasi assente nella documentazione successiva con l'affermarsi di carte in cui si indica la quantità o il valore del prestito: cfr. Pia, 2014, pp. 43-44. Utili confronti in Gaulin, Menant, 1998, pp. 37-67; Menant, Redon, 2004; cfr. anche Davide, 2003, pp. 639-668. Un'analisi in prospettiva civilistica è offerta da Volante, 2012 e Conetti, 2017. 
supporre che la commissione iniziale venisse sommata al capitale prestato, come suggerisce un atto del 1244 relativo all'impegno da parte del capitolo cattedrale a restituire un prestito ricevuto nel 1240 dall'operatore savonese Bonifacio Bucaordei, somma a suo tempo ottenuta per far fronte a un precedente debito con l'astigiano Raimondo Pelletta ${ }^{16}$.

Il notaio descrive con accuratezza l'origine delle somme oggetto di transazione: nel documento del 1240 che certifica l'instaurarsi del rapporto, si fa riferimento alla somma di 100 lire che, nell'impegno a restituire del 1244, è definita pecunia pretaxata ${ }^{17}$. II verbo taxare - usato come espressione tecnica in alcune restituzioni di male ablata per definire la penalità che i vicari applicano sulle usure confessate ${ }^{18}$ - nel caso del contratto tra il capitolo e il prestatore savonese sembra funzionale a individuare il costo dell'operazione, evidentemente fissato in precedenza, come rivela il prefisso "pre". D'altro canto il fatto che i due documenti, rogati a distanza di quattro anni l'uno dall'altro, riportino la stessa cifra di 100 lire, senza segnalare corresponsioni parziali da parte del debitore, è di per sé indicativo dell'assorbimento dell'interesse nella carta di mutuo iniziale, nella quale la somma era stata appunto pretaxata. L'esplicita dichiarazione del ricorso al credito di Bucaordei per far fronte a impegni non onorati con Pelletta segnala inoltre una condizione di non immediata solvibilità del capitolo che non poteva non essere valutata in sede di negoziazione del prestito, con probabili aggravi sui costi dell'operazione.

In quale modo il notaio costruisce l'efficacia di questa convenzione mirata a "sfumare" il pagamento degli interessi? Tre clausole del formulario usato al momento della stipulazione del prestito nel 1240 sono utili per comprendere le scelte operate nella costruzione del rapporto creditizio:

- l' «exceptio non numerate pecunie et non recepte»;

- la dichiarazione da parte del capitolo che «pecuniam conversam esse totam in utilitatem ecclesie»;

- la garanzia da parte dei debitori «quod non sit (...) necesse probare pecuniam esse conversam in utilitatem ecclesie».

La prima eccezione, peraltro ricorrente nei formulari dei mutui, si presta a coprire un'eventuale corresponsione non completa della cifra dichiarata e nel caso specifico, combinandosi con le altre condizioni, costituisce un'efficace tutela per il prestatore ${ }^{19}$, che in questo modo elude la questione degli interessi verosimilmente computati nella cifra iniziale. La pretaxatio cui si fa cenno nel successi-

16 Vergano (ed.), 1942, doc. 27, pp. 23-24 (1240) e doc. 64, pp. 76-77 (1244);

17 Pia, 2014, pp. 47-48.

18 Pia, 2014, p. 97. Per una lettura approfondita, cfr. Gaulin, Todeschini, 2019. Per articolate e innovative puntualizzazioni storiografiche sul tema dell'usura, cfr. Todeschini, 2002; Todeschini, 2007; Todeschini, 2012, pp. 119-130.

19 Volante, 2012, pp. 39 sgg. 
vo impegno a pagare del 1244 copre dunque con molta probabilità la differenza tra la somma dichiarata e quella effettivamente concessa in prestito. L'uso dell' "exceptio non numerate pecunie et non recepte» per nascondere un credito inferiore alla cifra certificata - caricando sulla carta di mutuo i costi dell'operazione - sembra confermato dall'ambigua formulazione delle clausole successive in cui si precisa che tutto il denaro è devoluto «in utilitatem ecclesie» ma che tale destinazione non deve essere provata. Proprio quest'ultima dichiarazione rafforza l'ipotesi che Bucaordei intendesse porre la convenzione al riparo da eventuali contestazioni sulla composizione della cifra pattuita, nella quale presumibilmente erano compresi fin dall'inizio gli interessi e che pertanto, nonostante le affermazioni dei debitori, non era interamente conversa «in utilitatem ecclesie».

\section{3. "Aliqua alia instrumenta»: un debito definito in itinere}

Se il mutuo contratto dal capitolo con il prestatore savonese negli anni Quaranta del Duecento rivela la consapevole combinazione delle clausole formulari in un atto "iniziale", con lo scopo probabile di occultare i costi del credito, in altre operazioni è il ricorso a documenti distinti a consentire la definizione di un rapporto di dare-avere.

Alla fine del Duecento risalgono alcuni contratti tra il vescovo di Asti ed esponenti della famiglia Solaro - legata alla Chiesa e al vertice dello schieramento guelfo - relativi all'area di Govone, località dell'attuale Cuneese nella quale entrambe le parti erano radicate ${ }^{20}$. II 23 novembre 1289, viene stipulata una «emptio bonorum et iurium »: il presule Oberto acquista beni e diritti sul castello e sul villaggio, mentre il cedente, Raineri Solaro, rilascia quietanza per 800 lire $^{21}$; il 22 e il 23 giugno 1291, Solaro emette una nuova ricevuta per le 800 lire relative alla compravendita del 1289, ma alla somma sono aggiunte altre 600 lire $^{22}$. Questa ulteriore cifra è ricondotta dal rogatario, in modo piuttosto vago, al «precio dicte vendicionis ut (...) partes asserunt sive sit causa mutui sive quacumque ex causa»: viene pertanto dichiarato il rapporto con l'emptio di beni e diritti in Govone, ma non si precisa se le 600 lire traessero origine da un mutuo o da "altra causa». Non solo: si aggiunge che, «licet appareant aliqua alia instrumenta» nei quali Solaro rilascia quietanza

per aliquibus partibus supradicti precii, (...) propterea veritas est quod non habuit nisi integram solucionem (...) ita quod instrumenta ipsi Raynerio non faciant aliquod preiudicium in eo quod per ea ultra debitum habuisse reperiatur quia in veritate ipse Raynerius totum habuit quod habere debebat occasione predicte vendicionis ${ }^{23}$.

20 Castellani, 1998, pp. 65-70.

21 Assandria, 1904, doc. 121, pp. 267-272.

22 Assandria, 1904, doc. 122, pp. 272-274.

23 Ivi, p. 274. 
Un'excusatio non petita che mette in evidenza tre elementi:

- oltre alla carta relativa alle 600 lire, il cedente, Raineri Solaro, aveva emesso altre quietanze («aliqua alia instrumenta»);

- il compratore (il vescovo) garantisce che il pagamento è avvenuto in un'unica soluzione contestuale alla stipula del contratto;

- questa garanzia serve a evitare al venditore il preiudicium di aver percepito somme ultra debitum.

Osservando nel suo rovescio, per così dire, l'orditura degli atti, emerge una rateizzazione del dovuto cui si collega il sospetto che il venditore abbia ricevuto somme ultra debitum. II notaio formula dunque un documento volto a sancire e nello stesso tempo a smentire il pagamento rateale di immobili e diritti: in questo modo sono condotte ai limiti della contraddizione sia la valenza certificatoria dell'instrumentum sia la sua funzione di stabilire i termini dei contratti.

Sui diversi passaggi sopra ricordati, va definendosi la relazione di debito-credito tra le parti:

- le ricevute di 800 e 600 lire rilasciate da Solaro certificano che questi non ha più nulla da pretendere dal vescovo;

- la precisazione sulle ragioni della corresponsione del denaro - effettuata contestualmente da Solaro e dal rappresentante del presule - nella sua genericità ("causa mutui sive quacumque ex causa») costituisce per il vescovo un'ulteriore garanzia di aver pagato il dovuto e, per Solaro, un iniziale riconoscimento della correttezza dell'operazione;

- quanto sostiene il rappresentante del vescovo, infine, completa la legittimazione della prassi economica seguita da Solaro, poiché si afferma che il prezzo è stato corrisposto in un'unica soluzione, nonostante gli "aliqua alia instrumenta» e si aggiunge che tale contraddittoria dichiarazione è rilasciata affinché non derivi alcun pregiudizio a Solaro ${ }^{24}$.

\section{Cessione e rinegoziazione del credito}

L'elaborazione notarile può delineare percorsi di riassetto di relazioni creditizie di varia natura, assicurando sanzione giuridica a compromessi intercorsi tra le parti: è quanto emerge da un'operazione di inizio Trecento nella quale, ancora una volta, si incontrano rapporti distinti, facenti capo a circuiti mercantili e religiosi. Nel febbraio 1301, il notaio palatino Filippo Benengo roga due carte nella domus astigiana dei Predicatori ove vivono Andrea, vedova di Marchetto Cacherano, e la madre Acuisia ${ }^{25}$. Nel primo atto, dell'11 febbraio, Andrea dona ad Acuisia e al predicatore Rodolfo di Gorzano sei crediti ammontanti a circa 90 lire. All'ultimo

\footnotetext{
${ }^{24}$ L'operazione è analizzata in modo dettagliato in Pia, 2014, pp. 48-50.

${ }^{25}$ Dacquino, Cotto Meluccio, 1983, docc. 17 e 18, pp. 67-69. Una ricostruzione sugli insedianti dei Mendicanti è offerta da Bordone, 2003, pp. 515-533.
} 
tra i sei contratti riepilogati nella donazione - un credito verso Rolando Tuitor e il figlio di questi dal valore indicato di 20 lire -, si riferisce anche il secondo atto, una quietanza del 13 febbraio che rivela un intervento di negoziazione da parte dei cessionari, Acuisia e Rodolfo. In tale quietanza, il debito dei Tuitor è indicato in modo contraddittorio: da una parte, infatti, si fa cenno a una ricevuta iniziale dei Tuitor ammontante a 29 lire - somma che questi ultimi si erano impegnati 13 mesi prima a «exercere et merc[...] in suis mercandiis[...] licitis [...] cum sua parte lucri ad terminum in ipso instrumento compre[henso]» - dall'altra, la convenzione stipulata "precepto domine Andree» prevede che i Tuitor corrispondano ad Acuisia e al predicatore Rodolfo 20 lire, cioè la somma dichiarata nella donatio complessiva dell'11 febbraio e non le 29 lire indicate nella ricevuta.

La scrittura notarile fissa dunque una ricognizione - e una conseguente "stabilizzazione" - di crediti spettanti alla vedova Cacherano che vanno a costituire un fondo gestito dalla madre di questa e dal predicatore Rodolfo; l'esigibilità di uno di tali crediti richiede però un ulteriore intervento del rogatario e la rinegoziazione della cifra da restituire a favore dei debitori, i Tuitor. Si trattava verosimilmente di un investimento, affidato da Andrea ai Tuitor sotto forma di deposito ${ }^{26}$, nel quale la differenza tra la ricevuta e la quietanza corrisponde al guadagno previsto, 9 lire, pari al 45\%. È probabile che il passaggio del credito alla madre di Andrea e al Predicatore abbia comportato la scelta di esigere la somma senza pretendere il guadagno prestabilito; il titolo donato sembra cioè liberato dalla componente speculativa a favore di un rientro immediato della somma investita: un accordo - cui il notaio conferisce stabilità e publica fides - nel quale l'esigenza di liquidità dei creditori è soddisfatta lasciando l'eventuale profitto ai destinatari del deposito.

\section{Sperimentazioni nel contenzioso fiscale}

L'intervento dei notai per definire rapporti di debito-credito può assumere anche valore politico: un versante dell'opera di certificazione - giocata tra esigenze di equilibrio comunitario e dinamiche fiscali - che emerge dalla documentazione relativa a un titolo di proprietà trasferito a seguito di un procedimento di datio in solutum connotato da una strutturale interferenza rispetto alle relazioni sociali ${ }^{27}$. L'atto, risalente al 1281, riguarda la restituzione da parte del comune di Asti a Girardo Piazza di quote del fodro che egli aveva anticipato all'istituzione cittadina. Quest'ultima sana la propria esposizione trasferendo a Piazza i diritti sul debito degli eredi di Oberto di Grana verso il comune, debito derivato dalla mancata corresponsione del fodro da parte di Oberto, in occasione della stessa imposizione cui aveva contribuito Piazza anticipando le quote sopra citate.

${ }^{26}$ Utili prospettive in Calleri, Puncuh, 2002, pp. 273-376, cfr. anche Massetto, 2002, pp. 251-327.

27 Cotto Meluccio, Fissore, Gosetti, Rossanino (edd.), 1986, doc. 175, pp. 248-252. 
Poiché i di Grana non versano quanto stabilito, Giacomo di San Miniato, giudice e vicario comunale, previa valutazione degli extimatores del comune, concede a Piazza la datio in solutum su terreni dei di Grana per un valore pari alla loro esposizione, con l'aggiunta dell'interesse di 3 denari ogni $2^{28}$. In base a capitula e ordinamenta, resta però ai di Grana la facoltà di rientrare nella proprietà dei loro fondi, a condizione di restituire entro 10 anni a Piazza il dovuto applicando un tasso di 5 denari ogni 3: se questo non accadesse, scaduto il termine decennale, i beni resteranno a Piazza ${ }^{29}$.

Analogamente al caso dei crediti della vedova Cacherano analizzato in precedenza, anche in questo rapporto tripartito tra il comune, Piazza e i di Grana, il notaio effettua una sorta di certificazione riepilogativa che definisce le relazioni di debito-credito. Nella pattuizione tra Piazza e il comune, tuttavia, l'intervento notarile presenta tratti di sperimentalità e agisce in una sorta di zona grigia in cui la distinzione tra pubblico e privato sfuma decisamente. La compensazione del credito di Piazza infatti si basa su una convenzione per le cui clausole è previsto l'inserimento nella legislazione comunale; nelle more dell'adozione come norma statutaria, le condizioni sono sancite dall'atto del notaio che non a caso ribadisce la validità dell'accordo anche qualora non entrasse stabilmente negli ordinati cittadini:

et si [capitulum] positum non fuerit vel positum removeretur, nichilominus tamen presens instrumentum observari debeat ut capitulum speciale non obstante capitulo de iusticia facienda et non obstante capitulo de racione facienda et generaliter non obstantibus capitulis consilii publicis vel privatis, factis vel faciendis, que huic instrumento in aliquo obviarent ${ }^{30}$.

La scrittura notarile dà forma, dunque, all'elaborazione di un procedimento nuovo di gestione del contenzioso fiscale, impostando un meccanismo che trasferisce le situazioni di insolvenza ai privati e garantisce al comune un'entrata immediata, mentre i cessionari dei diritti di esazione - che lucrano un interesse di 3 denari ogni 2 dovuti - si rivalgono sui beni dei contribuenti morosi. L'interferenza rispetto all'assetto patrimoniale degli insolventi ha ricadute sui rapporti sociali, come rivela la tutela della quale possono fruire i proprietari dei beni oggetto dalla datio in solutum: la rateizzazione decennale del debito, sia pure con l'ulteriore grave penalità di 5 denari ogni 3 dovuti. Un percorso restituito dalla complessa composizione dell'atto, entro il quale l'opera del notaio non si limita ad assicurare la publica fides ai rapporti, ma pone efficaci basi giuridiche per formule innovative nella compensazione del debito verso il comune.

\footnotetext{
${ }^{28}$ Sulla datio in solutum, cfr. Saccoccio, 2007, pp. 4893-4911; Sinisi, 2003, pp. 1032, 1036. Sull'ufficio degli estimatori, cfr. Vallerani, 2000, p. 16.

${ }^{29}$ Castellani, 1998, pp. 122, 144. Sulla normativa statutaria relativa agli interessi richiesti ai debitori insolventi, cfr. Bordone, 2003, p. 525.

${ }^{30}$ Cotto Meluccio, Fissore, Gosetti, Rossanino (edd.), 1986, doc. 175, pp. 251-252.
} 


\section{Conclusioni}

Appare evidente come l'intervento del notaio definisca elementi di snodo delle relazioni economiche, delle quali coglie le criticità che la scrittura tenta di risolvere stemperando i tratti di incertezza, costruendo meccanismi di compensazione e di regolazione, e ancora elaborando modi e forme dell'incontro tra credito e politica nel caso dell'inadempienza fiscale.

D'altro canto Rolandino Passaggeri assimila l'opera del notaio alla custodia di un tesoro costruito su fides e legalitas:

tabelliones vel scrinearios appellamus, eo quod sicut inventum thessaurum in scrineo reconditur ut servetur, sic merito debet omnis fides omnisque legalitas et quicquid eis in fide ac legalitate committitur per eos inviolabiliter reservari ${ }^{31}$.

Non solo: "omnibus que scripserint fides datur, nec audet quis de levi ausu temerario infringere publice quod scripserint $»^{32}$. La fides fissata in un atto non può dunque essere «infranta»: in questa prospettiva mediante la scrittura si va costruendo un "tesoro" che inquadra rapporti connotati da uno spiccato dinamismo e che - proprio per rispondere alla loro strutturale instabilità - assume a sua volta tratti dinamici, al fine di regolare la proiezione nel tempo di obbligazioni e impegni.

Tale percorso è evidente nella complessa compensazione del debito fiscale, incentrata sia sul principio generale di una restituzione dilatata nel tempo che tuteli le parti coinvolte sia sulla possibile validità una tantum del meccanismo di compensazione qualora non fosse inserito nei capitula statutari. Tuttavia anche negli atti che chiariscono o ribadiscono interessi e costi di operazioni finanziarie è in gioco la durevole funzionalità di rapporti potenzialmente conflittuali, poiché nell'atto notarile non è semplicemente sancita la rinegoziazione ma sono altresì impostati gli strumenti che ne assicurano l'efficacia in prospettiva diacronica.

Si tratta di pratiche articolate che corrispondono a precise competenze in capo al notaio, il quale, secondo il diffuso formulario duecentesco del giurista Martino da Fano, è dotato della peculiare capacità di discernere: «discretus, in intelligendo, disquirendo et componendo ${ }^{33}$. Comprendere le esigenze dei contraenti (intelligere), verificarne i fondamenti (disquirere), dare loro forma legittima (componere) costituiscono i fondamenti dell'opera di certificazione del notaio, in quanto depositario della publica fides. Quest'ultima, tuttavia, richiede di essere collocata nella concretezza di sistemi di relazione spesso ambigui e conflittuali, come suggerisce Attilio Bartoli Langeli:

la fides delle scripturae dei notai è (...) rappresentata come una realtà limpida, monolitica, assoluta. II nostro compito, invece, è di distinguere e scomporre. E

\footnotetext{
${ }^{31}$ Gaudenzi (ed.), 1896, p. 58.

32 Ivi.

${ }^{33}$ Piergiovanni, 2012b, p. 1462.
} 
si vedrà che la publica fides, lungi dall'essere un'idea platonica, è cosa di questo mondo, che partecipa delle situazioni storiche, le determina da par suo ma ne subisce anche i condizionamenti ${ }^{34}$.

È quanto ci viene confermato dalla sistematica registrazione di relazioni economiche che si è sommariamente esaminata: la publica fides in effetti si conferma "cosa di questo mondo" sia nella fissazione di rapporti standardizzati di debito-credito - sempre influenzati dai percorsi di riassetto costruiti dai notai - sia, in misura maggiore, negli atti nei quali le spinte contrastanti di pratiche finanziarie e normative canoniche, di usi e consuetudini si intersecano mettendo in evidenza nodi problematici che la scrittura notarile tenta di superare «intelligendo, disquirendo et componendo».

\section{Bibliografia}

Assandria G. (ed.) 1904: /l Libro Verde della Chiesa d'Asti, I, Pinerolo, Tip. Chiantore-Mascarelli

Bartoli Langeli A., 2004a: Prefazione a Notai e chiese. Le istituzioni ecclesiastiche e religiose e la loro documentazione in Italia dal XII al XV secolo, in "Quaderni di storia religiosa", 11, 2004, pp. 7-13

Bartoli Langeli A., 2004b: "Scripsi et publicavi". II notaio come figura pubblica, I'instrumentum come documento pubblico, in Michetti R. (ed.), Notai, miracoli e culto dei santi. Pubblicità e autenticazione del sacro tra XII e XV secolo. Atti del Seminario Internazionale, Roma 5-7 dicembre 2002, Milano, Giuffrè, pp. 55-71

Belloni C., Lunari M., 2004: Introduzione a Belloni C., Lunari M. (edd.), I notai della curia arcivescovile di Milano (secoli XIV-XVI). Repertorio coordinamento di G. Chittolini, Roma, Ministero per i beni e le attività culturali, Direzione generale per gli archivi, pp. XI-LXXXIV

Bordone R., 1980: Città e territorio nell'alto medioevo: la società astigiana dal dominio dei Franchi all'affermazione comunale, Torino, Deputazione subalpina di storia patria

Bordone R. (ed.), 1994a: L'uomo del banco dei pegni. "Lombardi" e mercato del denaro nell'Europa medievale, Torino, Scriptorium

Bordone R., 1994b: I 'lombardi' in Europa. Primi risultati e prospettive di ricerca, in "Società e storia", 17, 63, pp. 1-17

Bordone R., 2003: Prime attestazioni degli ordini mendicanti nei comuni di Asti e di Vercelli, in "Bollettino storico-bibliografico subalpino", 101, 2, pp. 515-533

Bordone R., 2005: I Lombardi in Europa: uno sguardo d'insieme, in Bordone R., Spinelli F. (eds.), Lombardi in Europa nel Medioevo, Milano, Franco Angeli, pp.

\footnotetext{
${ }^{34}$ Bartoli Langeli, 2004b, p. 60.
} 


\section{9-39}

Calleri M., Puncuh D., 2002: Il documento commerciale in area mediterranea, in Magistrale F., Drago C., Fioretti P. (edd.), Libri, documenti epigrafi: possibilità di studi comparativi. Atti del Convegno internazionale di studio dell'Associazione italiana dei paleografi e diplomatisti, Bari, 2-5 ottobre 2000, Spoleto, CISAM, pp. 273-376

Cammarosano P., 1991: Italia medievale. Struttura e geografia delle fonti scritte, Roma, La Nuova Italia Scientifica

Castellani L., 1998: Gli uomini d'affari astigiani. Politica e denaro tra Piemonte ed Europa, Torino, Paravia Scriptorium

Chironi G., 2005: La mitra e il calamo. II sistema documentario della Chiesa senese in età pretridentina, secoli XIV-XVI, Roma, Ministero per i Beni culturali e ambientali, Dipartimento per i Beni archivistici e librari, Direzione generale per gli archivi

Chittolini G., 1994, "Episcopalis Curie Notarius". Cenni sui notai di curie vescovili nell'Italia centro-settentrionale alla fine del medioevo, in Società, istituzioni, spiritualità. Studi in onore di Cinzio Violante, Spoleto, CISAM, pp. 221-232

Conetti M., 2017: Economia e diritto nel Trecento. La repetitio di Niccolò Matarelli sul tema dell'interesse, Roma Istituto storico italiano per il Medio Evo

Cotto Meluccio A.M., Fissore G.G., Gosetti P., Rossanino E. (edd.), 1986: Le carte dell'Archivio Capitolare di Asti (secc. XII-XIII), Torino, Deputazione subalpina di storia patria

Dacquino P., Cotto Meluccio A.M., 1983: Carte astigiane del secolo XIV, Asti, Cassa di Risparmio di Asti

Davide M., 2003: I/ credito in Friuli nel Trecento, in "Studi Medievali", 3a serie, 44, 2, pp. 639-668

Fissore G.G., 1973: Problemi della documentazione vescovile astigiana per i secoli $X-X I$, in "Bollettino storico-bibliografico subalpino", 71, 2, pp. 416-510

Fissore G.G., 1977: Autonomia notarile e organizzazione cancelleresca nel comune di Asti. I modi e le forme dell'intervento notarile nella costruzione del documento medievale, Spoleto, CISAM

Fissore G.G., 1995: I documenti cancellereschi degli episcopati subalpini: un'area di autonomia culturale fra la tradizione delle grandi cancellerie e la prassi notarile, in Haidacher Ch., Köfler W. (edd.), Die Diplomatik der Bischofurkunde vor 1250. Referate zum VIII Internationalen Kongress für Diplomatik (Innsbruck 27. September-3. Oktober 1993), Innsbruck Tiroler Landesarchiv, pp. 281-304

Fissore G.G., 2003: lacobus Sarrachus notarius et scopolanus Astensis ecclesie: i chierici notai nella documentazione capitolare e vescovile ad Asti tra XIII e XIV secolo, in Puncuh D. (ed.), Studi in memoria di Giorgio Costamagna (19162000), Genova, Società Ligure di Storia Patria, pp. 365-414 
Fissore G.G., 2009: Protocolli e notai del Capitolo cattedrale nella seconda metà del secolo XIV. Introduzione, in Fissore G.G., Molina B., Scarcia G., I protocolli notarili dell'archivio capitolare di Asti (seconda metà del secolo XIV). Regesti, Torino Deputazione subalpina di storia patria, pp. VIII-XV

Fissore G.G., 2013: Tessere di un mosaico. II notariato ecclesiastico in Asti a partire dall'edizione di un frammento di manuale notarile dell'Archivio capitolare della Cattedrale, in Fissore G.G., Molina B., Pia E.C. (edd.), "Con l'augurio che il mestiere di studioso sia causa di gioia». Atti della Giornata di Studio in memoria di Renato Bordone, Asti, Centro studi Renato Bordone sui Lombardi, sul credito e sulla banca, pp. 25-59

Gaudenzi A. (ed.), 1896: Statuti delle Società di Popolo di Bologna, II, Società delle arti, Roma, Forzani

Gaulin J.-L., Menant F., 1998: Credit rural et endettement paysan dans I'Italie communale, in Berte M. (ed.), Endettement paysan \& credit rural dans l'Europe medievale et moderne. Actes des XVII Journees internationales d'histoire de l'Abbaye de Flaran, septembre 1995, Toulouse, Presses universitaires du Mirail, pp. 37-67

Gaulin J.-L., Todeschini G. (edd.), 2019: Male ablata. La restitution des biens mal acquis. XIIe-XVe siècles, Roma, École française de Rome

Massetto G.P., 2002: Osservazioni in materia di contratti nella Summa totius artis notarie, in Tamba G. (ed.), Rolandino e l'ars notaria da Bologna all'Europa. Atti del Convegno internazionale di Studi storici sulla figura e l'opera di Rolandino (Roma, 9-10 ottobre 2000), Milano, A. Giuffrè, pp. 251-327

Menant F., Redon O., 2004: Notaires et crédit dans l'Occident méditerranéen médiéval, Roma, École française de Rome

Olivieri A., 2003: Per la storia dei notai chierici nel Duecento: il caso del Piemonte, in Puncuh D. (ed.), Studi in memoria di Giorgio Costamagna (1916-2000), Genova, Società Ligure di Storia Patria, pp. 701-738

Pia E.C., 2010: I registri del chierico notaio astigiano Giacomo Saracco: principali tipologie documentarie per la definizione di relazioni economiche (1285-1316), in "Mélanges de l'École française de Rome, Moyen Âge", 122, 10, pp. 119-125

Pia E.C., 2013, Lombardi in Europa: una lettura storiografica, in "Rivista Storica Italiana", 125, 3, pp. 817-840

Pia E.C., 2014: La giustizia del vescovo: società, economia e Chiesa cittadina ad Asti tra XIII e XIV secolo, Roma, Viella

Pia E.C., 2017: Asti, Spoleto, CISAM

Piergiovanni V., 2012a: Martino da Fano e lo sviluppo del diritto notarile, in Piergiovanni V., Norme, scienza e pratica giuridica tra Genova e l'Occidente medievale e moderno, Genova, Società Ligure di Storia Patria, pp. 1455-1464

Piergiovanni V., 2012b: Notariato e rivoluzione commerciale: l'esempio di 
Rolandino, in Piergiovanni V., Norme, scienza e pratica giuridica tra Genova e l'Occidente medievale e moderno, Genova, Società Ligure di Storia Patria, pp. 1417-1426

Puncuh D. (ed.), 1966: Gli statuti del collegio dei notai genovesi nel secolo XV, in Miscellanea di storia ligure in memoria di Giorgio Falco, Genova, Istituto di Paleografia e storia medievale, pp. 267-310

Rolandini, 1565: Summa Artis notariae, Lugduni, apud I. Huguetan

Saccoccio A., 2007: Datio in solutum: storia e dogmatica di una locuzione, in Studi in onore di Luigi Labruna, Napoli, Editoriale Scientifica, pp. 4893-4911

Sinisi L., 2003: Un frammento di formulario notarile genovese del Trecento, in Puncuh D. (ed.), Studi in memoria di Giorgio Costamagna (1916-2000), Genova, Società Ligure di Storia Patria, pp. 1027-1046

Todeschini G., 2002: I mercanti e il tempio. La società cristiana e il circolo virtuoso della ricchezza fra Medioevo ed Età Moderna, Bologna, il Mulino

Todeschini G., 2007: Visibilmente crudeli. Malviventi, persone sospette e gente qualunque dal Medioevo all'Età moderna, Bologna, il Mulino

Todeschini G., 2012: Usury in Christian Middle Ages. A Reconsideration of the Historiographical Tradition (1949-2010), in Ammannati F. (ed.), Religione $e$ istituzioni religiose nell'economia euoropea, 1000-1800. Atti della XLIII settimana di studi della Fondazione Istituto internazionale di storia economica F. Datini (Prato, 8-12 maggio 2011), Firenze, Firenze University Press, pp. 119-130

Vallerani M., 2000: Le scritture di debito negli statuti dei comuni italiani, in Credito e società: le fonti, le tecniche, gli uomini, secc. XIV-XVI. Atti del convegno internazionale di studi (Asti-Chambery, 24-27 settembre 1998), Asti, Tipografia Astese, pp. 9-22

Varanini G.M., Gardoni G., 2009: Notai vescovili del Duecento tra curia e città, in Piergiovanni V. (ed.), II notaio e la città. Essere notaio: i tempi e i luoghi (secoli XII-XV). Atti del Convegno di studi storici (Genova, 9-10 novembre 2007), Milano, Giuffrè, pp. 241-272

Vergano L. (ed.), 1942: Le carte dell'Archivio Capitolare di Asti (1238-1272), Torino, [s.n.], 1942 (Casale Monferrato, Miglietta, Milano \& C.)

Volante R. 2012: Il mutuo nel diritto comune: il problema del valore finanziario dai glossatori a Pothier, Napoli, Jovene 
